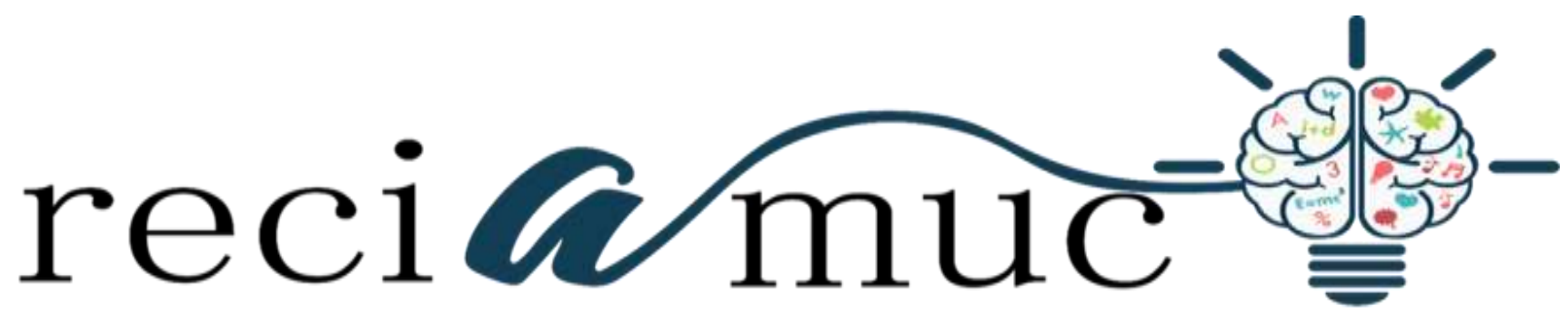

Revista científica de investigación actualización del mundo de las ciencias

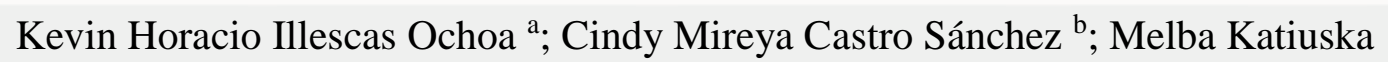
Carrera Saltos c; Martín Federico García Arteaga d

Insulinoterapia en pacientes con diabetes mellitus tipo 2

When to begin insulinotherapy in patients with mellitus type 2 diabetes

Revista Científica de Investigación actualización del mundo de las Ciencias. Vol. 3 núm., 2, abril, ISSN: 2588-0748, 2018, pp. 875-894

DOI: $10.26820 / \mathrm{reciamuc/3.(2).abril.2019.875-894}$

URL: http://reciamuc.com/index.php/RECIAMUC/article/view/370

Código UNESCO: 3205 Medicina Interna

Tipo de Investigación: Artículo de Revisión

(C) RECIAMUC; Editorial Saberes del Conocimiento, 2019

Recibido: 15/01/2019

Aceptado: 07/02/2019

Publicado: 01/04/2019

Correspondencia: kevin.illescas@gmail.com

a. Médico; Investigador Independiente; Guayaquil, Ecuador; kevin.illescas@ gmail.com

b. Médico; Investigador Independiente; Guayaquil, Ecuador; cindycastros@ hotmail.com

c. Master Universitario en Investigación Biomédica, Médico; Investigador Independiente; Guayaquil, Ecuador; melpandita_forever@hotmail.com

d. Médico; Investigador Independiente; Guayaquil, Ecuador; nitramgarcia13@ hotmail.com 


\section{Insulinoterapia en pacientes con diabetes mellitus tipo 2}

Vol. 3, núm. 2., (2019)

Kevin Horacio Illescas Ochoa; Cindy Mireya Castro Sánchez; Melba Katiuska Carrera Saltos; Martín Federico García Arteaga

\section{RESUMEN}

La insulina, la molécula central en la diabetes, sigue siendo el mejor tratamiento para la enfermedad, incluso después de ochenta y cinco años de uso. Aunque es asociada con varios mitos y aprensiones, la terapia con insulina no tiene contraindicaciones específicas. El objetivo es implementar insulina que imite la secreción fisiológica de insulina, debido a que las personas con diabetes mellitus tipo II realizan una producción insuficiente de la molecula. La administración de insulina exógena basal es esencial para regular la descomposición del glucógeno, la gluconeogénesis, la lipólisis y la cetogénesis. El régimen de insulina incluye regímenes de insulina de múltiples componentes, inyecciones diarias múltiples (MDI) como sus siglas en inglés o dispositivos de infusión de insulina. Las especies y la dosis utilizadas deben ser consistente y la técnica de inyección del paciente debe revisarse periódicamente con la diabetes y el equipo de atención. El uso efectivo de la insulina para obtener el mejor control metabólico requiere una comprensión de la duración de la acción de varios tipos de insulina y la relación de los niveles de glucosa en sangre con el ejercicio, la ingesta de alimentos, las enfermedades intercurrentes, ciertos medicamentos y estrés para aprender a ajustar la dosis de insulina adecuadas con la finalidad de lograr los objetivos individualizados establecidos entre el paciente, la familia y equipo de atención de diabetes.

Palabras clave: Diabetes; Diabetes mellitus tipo II; Insulina; insulina terapia. 


\title{
Insulinoterapia en pacientes con diabetes mellitus tipo 2
}

Vol. 3, núm. 2., (2019)

Kevin Horacio Illescas Ochoa; Cindy Mireya Castro Sánchez; Melba Katiuska Carrera Saltos; Martín Federico García Arteaga

\begin{abstract}
Insulin, the central molecule in diabetes, remains the best treatment for the disease, even after eighty-five years of use. Although it is associated with several myths and apprehensions, insulin therapy has no specific contraindications. The goal is to implement insulin that mimics the physiological secretion of insulin, because people with type II diabetes mellitus produce insufficient insulin production. The administration of exogenous basal insulin is essential to regulate the breakdown of glycogen, gluconeogenesis, lipolysis and ketogenesis. The insulin regimen includes multiple component insulin regimens, multiple daily injections (MDI) as its initials in English or insulin infusion devices. The species and dose of insulin should be consistent and the patient's injection technique should be reviewed with diabetes and the care team. The effective use of insulin to obtain the best metabolic control requires an understanding of the duration of action of various types of insulin and the relationship of blood glucose levels with exercise, food intake, intercurrent diseases, medication and stress symptoms to learn how to adjust the appropriate insulin dose with the determination to achieve the individualized goals set between the patient, the family and the diabetes care team.
\end{abstract}

Key words: Diabetes; Type II diabetes mellitus; Insulin; Insulin therapy. 


\section{Insulinoterapia en pacientes con diabetes mellitus tipo 2}

Vol. 3, núm. 2., (2019)

Kevin Horacio Illescas Ochoa; Cindy Mireya Castro Sánchez; Melba Katiuska Carrera Saltos; Martín Federico García Arteaga

\section{Introducción.}

La insulina es secretada continuamente por las células beta de manera dependiente de la glucosa durante todo el día. También se secreta en respuesta a las cargas de carbohidratos orales, incluida una gran liberación de insulina en la primera fase que suprime la producción de glucosa hepática, seguida de una liberación más lenta de insulina en la segunda fase que cubre los carbohidratos ingeridos. (Ritzel \& Bulter, 2002)

La diabetes mellitus tipo 2 se asocia con resistencia a la insulina y falla de células beta lentamente progresiva. Para cuando la diabetes tipo 2 se diagnostica en pacientes, hasta la mitad de sus células beta no funcionan correctamente. La falla de las células beta continúa a una tasa de aproximadamente 4 por ciento cada año. Por lo tanto, los pacientes con diabetes tipo 2 a menudo se benefician de la terapia con insulina en algunos casos. (California, 2010)

Las terapias con insulina crean ciertas preocupaciones en pacientes y médico tratante, el dolor, el aumento de peso y la hipoglucemia pueden ocurrir con la terapia con insulina. El dolor está asociado con terapia de inyección y control de glucosa, aunque hoy en día existen agujas más delgadas y más cortas que ayudan a disminuir el dolor. El aumento de peso asociado con la terapia con insulina se debe a los efectos anabólicos de la insulina, aumento del apetito, alimentación defensiva por hipoglucemia y aumento de la retención de calorías relacionado con disminución de la glucosuria.

En el Estudio Prospectivo de Diabetes del Reino Unido, los pacientes con diabetes tipo 2 que estaban tomando insulina ganaron un promedio de $8 \mathrm{lb}, 13 \mathrm{oz}$ (4 kg), lo que se asoció con una 


\section{Insulinoterapia en pacientes con diabetes mellitus tipo 2}

Vol. 3, núm. 2., (2019)

Kevin Horacio Illescas Ochoa; Cindy Mireya Castro Sánchez; Melba Katiuska Carrera Saltos; Martín Federico García Arteaga

disminución del 0.9 por ciento en el nivel de A1C en comparación con los pacientes en tratamiento convencional. (Lanceta, 2010)

La hipoglucemia puede ocurrir por un desajuste entre la ingesta de insulina y carbohidratos, el ejercicio o el consumo de alcohol. La hipoglucemia se ha asociado con un mayor riesgo de demencia y puede tener implicaciones en la arritmia cardíaca. Todos los pacientes deben recibir instrucciones sobre los síntomas y tratamiento de hipoglucemia.

Las pautas de la Asociación de Diabetes (ADA) recomiendan que se verifique el nivel de glucosa en sangre si se sospecha hipoglucemia (glucosa nivel inferior a $70 \mathrm{mg}$ por $\mathrm{dL}$ mmol por L), luego tratado con carbohidratos de acción, como jugo o tabletas de glucosa. El nivel de glucosa en sangre se debe volver a comprobar después de 15 minutos para asegurarse de que se haya normalizado. (ADA, 2004)

Un estudio epidemiológico ha generado preocupación sobre el riesgo de cáncer con la glargina (Lantus) y otras terapias con insulina. La teoría de la glargina es más probable que cause cáncer debido a su alta afinidad por el factor de crecimiento similar a la insulina tipo I receptor. Una declaración de consenso de la ADA indica que este posible riesgo necesita más investigación, pero no debería ser una limitación en la elección del tratamiento. (Jonasson \& Ljung, 2009)

\section{Métodos y materiales.}

Para el desarrollo de este proceso investigativo, se plantea como metodología la encaminada hacia una orientación científica particular que se encuentra determinada por la necesidad de indagar en forma precisa y coherente una situación, en tal sentido (Davila, 2015) 


\section{Insulinoterapia en pacientes con diabetes mellitus tipo 2}

Vol. 3, núm. 2., (2019)

Kevin Horacio Illescas Ochoa; Cindy Mireya Castro Sánchez; Melba Katiuska Carrera Saltos; Martín Federico García Arteaga

define la metodología "como aquellos pasos previos que son seleccionados por el investigador para lograr resultados favorables que le ayuden a plantear nuevas ideas”. (p.66)

Lo citado por el autor, lleva a entender que el desarrollo de la acción investigativa busca simplemente coordinar acciones enmarcadas en una revisión bibliográfica con el fin de complementar ideas previas relacionadas Insulinoterapia en pacientes con diabetes mellitus tipo 2, a través de una revisión de literatura, para así finalmente elaborar un cuerpo de consideraciones generales que ayuden a ampliar el interés propuesto.

\section{Tipo de Investigación.}

Dentro de toda práctica investigativa, se precisan acciones de carácter metodológico mediante las cuales, se logra conocer y proyectar los eventos posibles que la determinan, así como las características que hacen del acto científico un proceso interactivo ajustado a una realidad posible de ser interpretada. En este sentido, se puede decir, que la presente investigación corresponde al tipo documental, definido por Castro (2016), "se ocupa del estudio de problemas planteados a nivel teórico, la información requerida para abordarlos se encuentra básicamente en materiales impresos, audiovisuales y /o electrónicos”. (p.41).

En consideración a esta definición, la orientación metodológica permitió la oportunidad de cumplir con una serie de actividades inherentes a la revisión y lectura de diversos documentos donde se encontraron ideas explicitas relacionadas con los tópicos encargados de identificar a cada característica insertada en el estudio. Por lo tanto, se realizaron continuas interpretaciones con el claro propósito de revisar aquellas apreciaciones o investigaciones propuestas por diferentes 


\section{Insulinoterapia en pacientes con diabetes mellitus tipo 2}

Vol. 3, núm. 2., (2019)

Kevin Horacio Illescas Ochoa; Cindy Mireya Castro Sánchez; Melba Katiuska Carrera Saltos; Martín Federico García Arteaga

investigadores relacionadas con el tema de interés, para luego dar la respectiva argumentación a los planteamientos, en función a las necesidades encontradas en la indagación.

\section{Fuentes Documentales.}

El análisis correspondiente a las características que predomina en el tema seleccionado, llevan a incluir diferentes fuentes documentales encargadas de darle el respectivo apoyo y en ese sentido cumplir con la valoración de los hechos a fin de generar nuevos criterios que sirven de referencia a otros procesos investigativos. Para (CASTRO, 2016) las fuentes documentales incorporadas en la investigación documental o bibliográfica, "representa la suma de materiales sistemáticos que son revisados en forma rigurosa y profunda para llegar a un análisis del fenómeno".(p.41). Por lo tanto, se procedió a cumplir con la realización de una lectura previa determinada para encontrar aquellos aspectos estrechamente vinculados con el tema, con el fin de explicar mediante un desarrollo las respectivas apreciaciones generales de importancia.

\section{Técnicas para la Recolección de la Información}

La conducción de la investigación para ser realizada en función a las particularidades que determinan a los estudios documentales, tiene como fin el desarrollo de un conjunto de acciones encargadas de llevar a la selección de técnicas estrechamente vinculadas con las características del estudio. En tal sentido, (Bolívar, 2015), refiere, que es "una técnica particular para aportar ayuda a los procedimientos de selección de las ideas primarias y secundarias”. (p. 71).

Por ello, se procedió a la utilización del subrayado, resúmenes, fichaje, como parte básica para la revisión y selección de los documentos que presentan el contenido teórico. Es decir, que 


\section{Insulinoterapia en pacientes con diabetes mellitus tipo 2}

Vol. 3, núm. 2., (2019)

Kevin Horacio Illescas Ochoa; Cindy Mireya Castro Sánchez; Melba Katiuska Carrera Saltos; Martín Federico García Arteaga

mediante la aplicación de estas técnicas se pudo llegar a recoger informaciones en cuanto a la revisión bibliográfica de los diversos elementos encargados de orientar el proceso de investigación. Tal como lo expresa, (Bolívar, 2015) "las técnicas documentales proporcionan las herramientas esenciales y determinantes para responder a los objetivos formulados y llegar a resultados efectivos" (p. 58). Es decir, para responder con eficiencia a las necesidades investigativas, se introdujeron como técnica de recolección el método inductivo, que hizo posible llevar a cabo una valoración de los hechos de forma particular para llegar a la explicación desde una visión general.

Asimismo, se emplearon las técnicas de análisis de información para la realización de la investigación que fue ejecutada bajo la dinámica de aplicar diversos elementos encargados de determinar el camino a recorrer por el estudio, según, (Bolívar, 2015) las técnicas de procesamiento de datos en los estudios documentales "son las encargadas de ofrecer al investigador la visión o pasos que debe cumplir durante su ejercicio, cada una de ellas debe estar en correspondencia con el nivel a emplear" (p. 123). Esto indica, que para llevar a cabo el procesamiento de los datos obtenidos una vez aplicado las técnicas seleccionadas, tales como: fichas de resumen, textual, registros descriptivos entre otros, los mismos se deben ajustar al nivel que ha sido seleccionado.

\section{Resultados}

Iniciando la terapia de insulina apropiada El Colegio Americano de Endocrinología y la Asociación Americana de Endocrinólogos Clínicos recomiendan el inicio de la terapia con insulina en pacientes con diabetes tipo 2 y un nivel inicial de A1C superior al 9\%, o si la diabetes no está controlada a pesar de ser óptima la terapia glucémica oral. (Jellinger \& Davidson, 2007) 


\section{Insulinoterapia en pacientes con diabetes mellitus tipo 2}

Vol. 3, núm. 2., (2019)

Kevin Horacio Illescas Ochoa; Cindy Mireya Castro Sánchez; Melba Katiuska Carrera Saltos; Martín Federico García Arteaga

La insulina se puede usar sola o en combinación con medicamentos orales, como la metformina (Glucofage). Esta recomendación se basa en la opinión de expertos y no en los resultados de la asignación al azar. Los ensayos controlados han comprobado diferentes enfoques en pacientes con un nivel inicial de A1C superior al 9 por ciento.

En un estudio realizado por (Lanceta, 2010) acerca del control intensivo temprano de la glucosa que comienza con una sulfonilurea, luego metformina, luego insulina se asoció con una reducción del $25 \%$ en complicaciones microvasculares y una reducción del riesgo del $12 \%$ en cualquier punto final relacionado con la diabetes, pero no se asoció con una reducción en la mortalidad por todas las causas.

Un subgrupo de pacientes asignados al azar terapia intensiva con metformina sola tuvieron un $36 \%$ de reducción en la mortalidad por todas las causas. (Lanceta., 2008) Esto respalda las pautas actuales de la ADA que recomiendan usar metformina como terapia farmacológica de primera línea; sin embargo, se deben agregar terapias adicionales si la diabetes no se controla con metformina sola.

Ensayos recientes han demostrado que el control intensivo de la glucosa (es decir, un objetivo de A1C de menos de 6.0 o 6.5 por ciento) no mejora y puede empeorar los resultados clínicos. Los pacientes mayores con una esperanza de vida limitada y los pacientes con un alto riesgo de hipoglucemia con enfermedad cardiovascular previa o enfermedad microvascular avanzada pueden beneficiarse de un control de glucosa menos estricto. (ADA, American Diabetes Association. Standards of medical care in diabetes, 2010) 


\section{Insulinoterapia en pacientes con diabetes mellitus tipo 2}

Vol. 3, núm. 2., (2019)

Kevin Horacio Illescas Ochoa; Cindy Mireya Castro Sánchez; Melba Katiuska Carrera Saltos; Martín Federico García Arteaga

El control de la glucosa, los efectos adversos, el costo, la adherencia, y la calidad de vida deben tenerse en cuenta al elegir un tipo de insulina. En general, la insulina análoga es similar a la insulina humana en el control de la diabetes, aunque algunos ensayos han encontrado niveles medios de A1C más altos en pacientes que toman insulina analógica en comparación con la insulina humana.

La insulina analógica generalmente causa menos hiperglucemia posprandial e hipoglucemia tardía. En un metanálisis reciente, el control glucémico no mejoró con la insulina análoga en comparación con la insulina humana, sino con la hipoglucemia nocturna que fue reducido. (Singh \& Ahmad, 2009)

Para elegir el tipo y los regímenes correctos de insulina se deben estudiar las necesidades y el estilo de vida del paciente y adaptarlos a estos parámetros. Una de las consideraciones más importantes es la farmacocinética de diferentes preparaciones de insulina ver (Figura $N^{\circ} 1$ y la Tabla $\mathrm{N}^{\circ}$ 1) en la tabla se define los términos de uso común en la terapia con insulina. (Endotext.org., 2010) 


\section{Insulinoterapia en pacientes con diabetes mellitus tipo 2}

Vol. 3, núm. 2., (2019)

Kevin Horacio Illescas Ochoa; Cindy Mireya Castro Sánchez; Melba Katiuska Carrera Saltos; Martín Federico García Arteaga

Figura $\mathbf{N}^{\mathbf{0}} 1$. Inicio de acción, pico y duración de preparaciones de insulina exógenas.

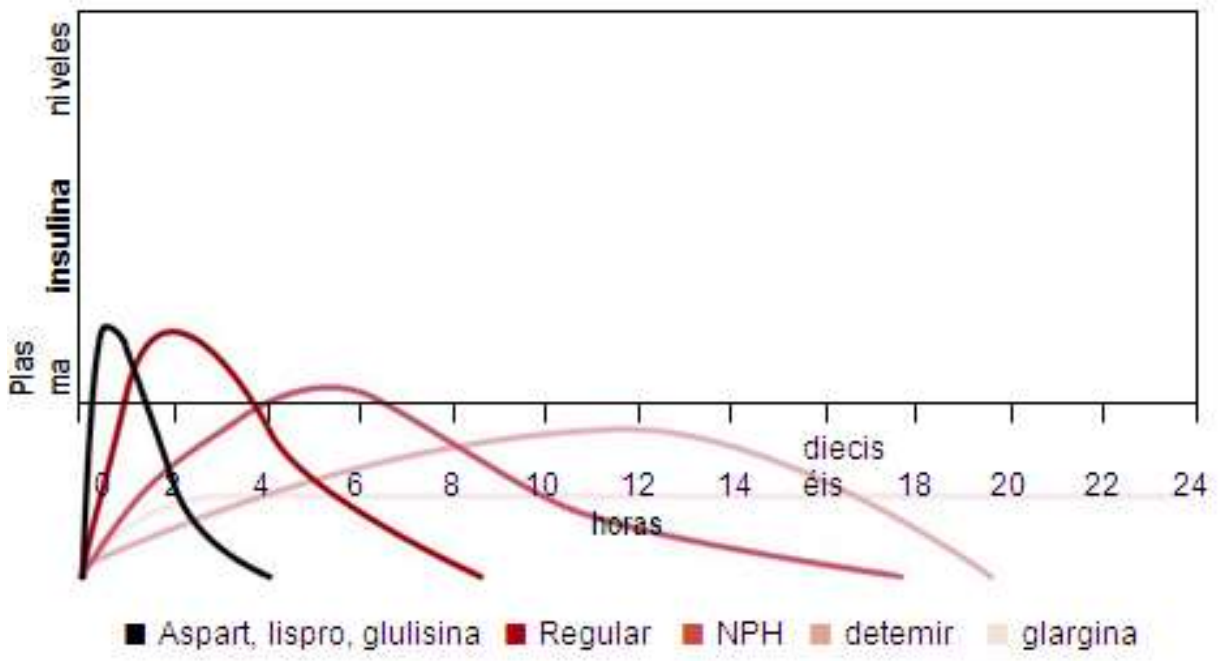

Fuente: (Endotext.org., 2010)

Tabla $\mathbf{N}^{\mathbf{0}} 1$. Términos de uso común en la terapia de insulina

\begin{tabular}{|c|c|c|}
\hline Termino & Definición & Cálculo \\
\hline Aumento & $\begin{array}{l}\text { Uso de insulina basal o de bolo para ayudar a mejorar el control de la } \\
\text { glucosa en pacientes con insuficiencia parcial de células beta }\end{array}$ & 0.3 unidad por $\mathrm{kg}$ \\
\hline Reemplazo & $\begin{array}{l}\text { Uso de insulina basal y en bolo para controlar la glucosa en sangre cuando } \\
\text { la producción de insulina endógena es mínima o ausente }\end{array}$ & 0.6 a 1.0 unidad por $\mathrm{kg}$ \\
\hline $\begin{array}{l}\text { Relación de } \\
\text { carbohidratos }\end{array}$ & $\begin{array}{l}\text { La cantidad de unidades de insulina necesarias para cubrir una cierta } \\
\text { cantidad de gramos de carbohidratos ingeridos }\end{array}$ & $\begin{array}{l}500 \text { dividido por el total de } \\
\text { insulina diaria } \\
\text { (generalmente alrededor de } \\
1 \text { unidad por } 10 \mathrm{~g} \text { ) }\end{array}$ \\
\hline $\begin{array}{c}\text { Corrección } \\
\text { (sensibilidad) }\end{array}$ & $\begin{array}{l}\text { ¿Cuánta unidad de insulina se espera que disminuya el nivel de glucosa en } \\
\text { sangre del paciente? cuando el nivel de glucosa en sangre está por encima } \\
\text { de los objetivos predefinidos, se puede agregar insulina de acción corta a la } \\
\text { dosis en bolo o administrarse por separado entre comidas }\end{array}$ & $\begin{array}{l}1.500 \text { dividido por el total } \\
\text { de insulina diaria } \\
\text { (generalmente alrededor de } \\
1 \text { unidad por } 25 \mathrm{~g} \text { ) }\end{array}$ \\
\hline
\end{tabular}

Fuente: (Endotext.org., 2010)

En un estudio realizado por (Holman \& Farmer, 2009) a pacientes que tenían diabetes tipo 2 no controlada y estaban tomando sulfonilurea y metformina fueron aleatorizados para recibir insulina premezclada, en bolo o análoga basal. Los niveles medios de A1C fueron similares entre 


\section{Insulinoterapia en pacientes con diabetes mellitus tipo 2}

Vol. 3, núm. 2., (2019)

Kevin Horacio Illescas Ochoa; Cindy Mireya Castro Sánchez; Melba Katiuska Carrera Saltos; Martín Federico García Arteaga

los grupos, pero la hipoglucemia fue más común en los grupos premezclados y en bolo, y el aumento de peso fue más común en el grupo en bolo. Los resultados de este estudio sugieren que agregar insulina basal a los antihiperglucémicos orales es similarmente efectivo, pero tiene menos efectos adversos en comparación con la adición de insulina premezclada o en bolo.

El objetivo de la insulina basal es suprimir la producción de glucosa hepática y mejorar la hiperglucemia en ayunas (Figura $\mathrm{N}^{\circ} 2$ ). Si la insulina basal se titula demasiado, también parcialmente cubra las comidas y provoque hipoglucemia durante la noche o si se pierde una comida. La insulina análoga de acción prolongada puede administrarse una o dos veces al día, dependiendo de la dosis. Las dosis más bajas pueden no durar 24 horas, mientras que las dosis más altas pueden impedir la absorción de insulina. El NPH se puede administrar de una a tres veces al día. El NPH es regularmente utilizado durante el embarazo y en pacientes que no pueden pagar el costo inicial de la insulina análoga. 
Insulinoterapia en pacientes con diabetes mellitus tipo 2

Vol. 3, núm. 2., (2019)

Kevin Horacio Illescas Ochoa; Cindy Mireya Castro Sánchez; Melba Katiuska Carrera Saltos; Martín Federico García Arteaga

Figura $\mathbf{N}^{\mathbf{a}}$ 2. Terapia de aumento con insulina basal. Perfil farmacocinético del uso de glargina, detemir o terapia con NPH una vez al día.

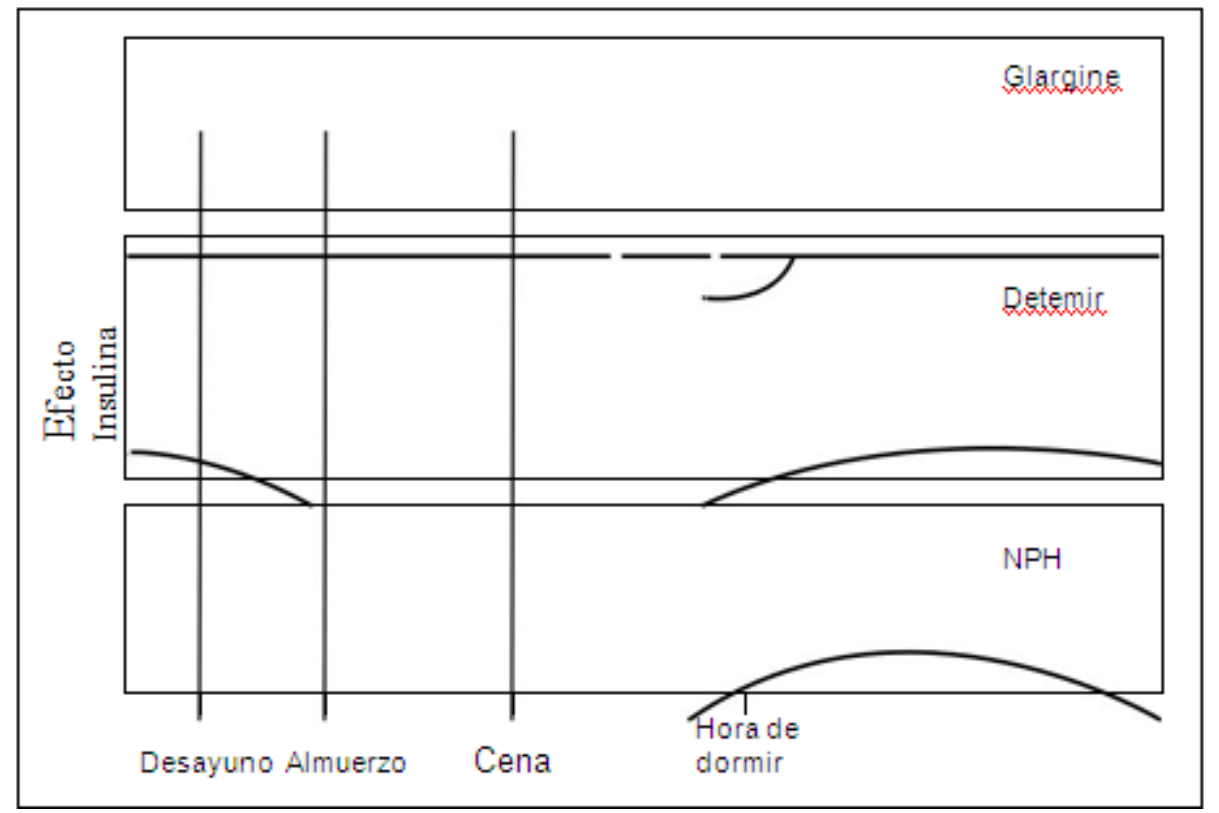

Fuente: (California, 2010).

La insulina en bolo también se puede usar para aumentar. La insulina de acción corta se administra antes de las comidas para cubrir las cargas de carbohidratos. La insulina análoga de acción corta se administra hasta 15 minutos antes de una comida para mantener los niveles de glucosa posprandiales de dos horas. Tomar insulina después de las comidas aumenta el riesgo de hiperglucemia posprandial temprana seguida de hipoglucemia tardía. En su lugar, se puede usar insulina regular y se administra de 30 a 45 minutos antes de las comidas. (Cobry \& McFann, 2010)

\section{Terapia de reemplazo}

La terapia de reemplazo incluye insulina de bolo basal y corrección o insulina premezclada.

Se debe considerar el reemplazo para pacientes con diabetes tipo 2 que no está controlado con 


\section{Insulinoterapia en pacientes con diabetes mellitus tipo 2}

Vol. 3, núm. 2., (2019)

Kevin Horacio Illescas Ochoa; Cindy Mireya Castro Sánchez; Melba Katiuska Carrera Saltos; Martín Federico García Arteaga

terapia de aumento y que pueden cumplir con dicho régimen o que desean un control más estricto.

La insulina en bolo se debe agregar a la insulina basal si se cumplen los objetivos de glucosa en ayunas, pero no los objetivos posprandiales. Cuando los niveles de glucosa en sangre están por encima de los objetivos predefinidos, se puede agregar insulina de acción corta adicional a la dosis en bolo antes de las comidas.

La insulina premezclada reduce de manera similar el A1C en comparación con la insulina de bolo basal NPH se combina con insulina regular o insulina análoga de acción corta y se administra dos o tres veces al día. Se necesitan menos inyecciones, pero los pacientes están más restringidos en sus hábitos alimenticios y horario. Los pacientes deben desayunar, almorzar, cenar y posiblemente comer alguna merienda a media mañana o algún refrigerio antes de acostarse para prevenir la hipoglucemia. Si se usa, la insulina correctora debe administrarse por separado con una insulina de acción corta. Esto puede aumentar el número de inyecciones en comparación con la terapia de bolo basal (Figura N³) (Miser \& Arakaki, 2010) 


\section{Insulinoterapia en pacientes con diabetes mellitus tipo 2}

Vol. 3, núm. 2., (2019)

Kevin Horacio Illescas Ochoa; Cindy Mireya Castro Sánchez; Melba Katiuska Carrera Saltos; Martín Federico García Arteaga

Figura No3. Terapia de insulina premezclada. Perfil farmacocinético del uso de insulina análoga de acción corta o insulina regular junto con NPH en un régimen de insulina premezclada

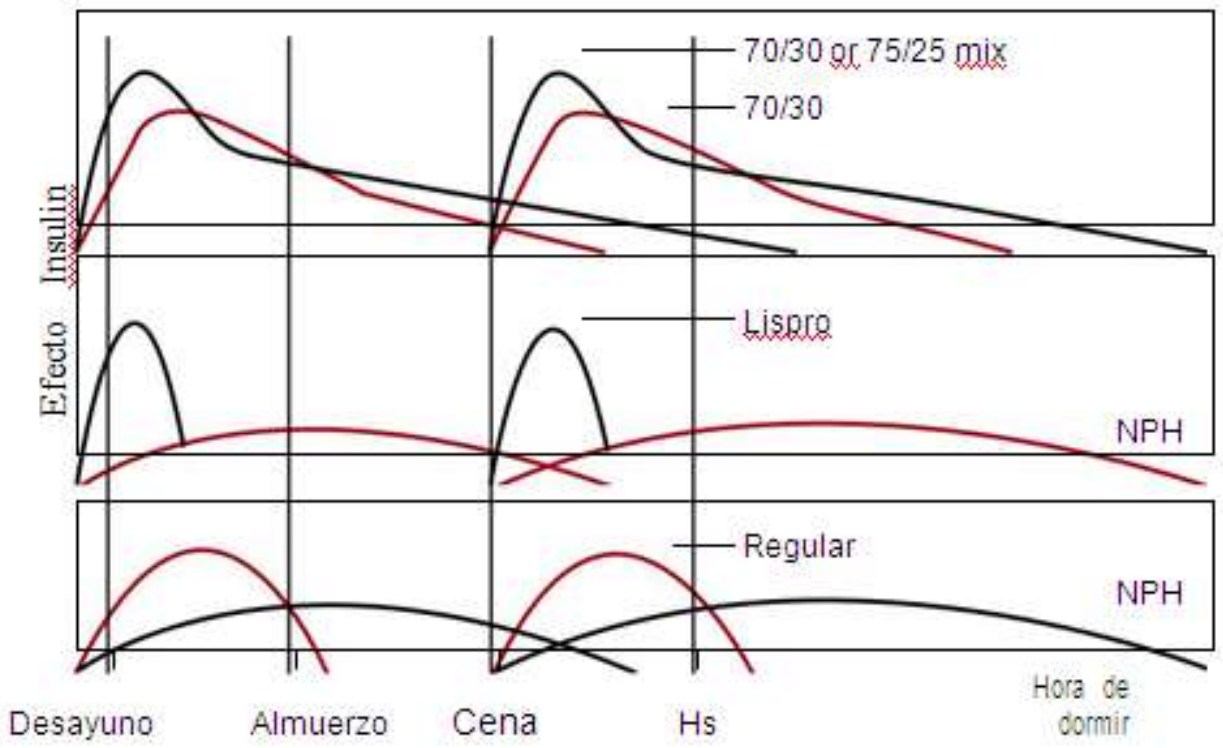

Fuente: (Miser \& Arakaki, 2010).

\section{Iniciación, titulación y seguimiento}

La dosis inicial de insulina es individualizada basada en la sensibilidad a la insulina del paciente. La terapia con insulina se puede comenzar con una dosis establecida, como 10 unidades de glargina al día, o mediante el uso de ecuaciones basadas en el peso. Ecuaciones para estimar aumento, reemplazo, carbohidratos relación y la terapia de corrección se enumeran en la Tabla $\mathrm{N}^{\mathrm{0}} 2$.

Cuando se usa la terapia de reemplazo, el 50 por ciento de la dosis de insulina diaria total se administra como basal y 50 por ciento como bolo, dividida antes del desayuno, almuerzo y cena.

La titulación de la insulina a lo largo del tiempo es crítica para mejorar el control glucémico y prevenir complicaciones relacionadas con la diabetes. Los objetivos actuales de ADA para el control de la glucosa se resumen en la Tabla $\mathrm{N}^{0} 2$. Las lecturas de glucosa en ayunas se 


\section{Insulinoterapia en pacientes con diabetes mellitus tipo 2}

Vol. 3, núm. 2., (2019)

Kevin Horacio Illescas Ochoa; Cindy Mireya Castro Sánchez; Melba Katiuska Carrera Saltos; Martín Federico García Arteaga

utilizan para valorar la insulina basal, mientras que las lecturas de glucosa preprandial y posprandial se usan para valorar la insulina a la hora de las comidas. (Riddle, 2003)

Tabla N². Asociación Americana de Diabetes. Objetivos de glucosa en sangre y A1C para pacientes con diabetes mellitus.

\begin{tabular}{|l|l|l|}
\hline Medición & Población general & Mujeres embarazadas \\
\hline Glucosa en sangre en & 90 a $130 \mathrm{mg}$ por dL & 60 a $100 \mathrm{mg}$ por dL $(3.33 \mathrm{a}$ \\
ayunas & $(5 \mathrm{a} 7,21 \mathrm{mmol}$ por L) & $5.55 \mathrm{mmol}$ por L) \\
Glucemia posprandial & $<180 \mathrm{mg}$ por dL & 100 a $130 \mathrm{mg}$ por dL (5.55 \\
& $(9,99 \mathrm{mmol}$ por L) & a $7.21 \mathrm{mmol}$ por L) \\
A1C & $<7.0$ por ciento & $<6.0$ por ciento \\
\hline
\end{tabular}

Fuente: (Riddle, 2003).

Los médicos pueden aumentar o disminuir la insulina basal y / o en bolo en un $10 \%$ según las lecturas de glucosa en el hogar del paciente. Algunos médicos han adoptado el cronograma de titulación del ensayo para tratar la insulina basal (Tabla 3). También es seguro y efectivo dar a los pacientes autonomía para ajustar la insulina por sí solos. Por lo general, la insulina aumenta o disminuye en 2 a 3 unidades cada tres a siete días si el nivel de glucosa en sangre del paciente no está dentro de los objetivos establecidos. (Riddle, 2003) 
Insulinoterapia en pacientes con diabetes mellitus tipo 2

Vol. 3, núm. 2., (2019)

Kevin Horacio Illescas Ochoa; Cindy Mireya Castro Sánchez; Melba Katiuska Carrera Saltos; Martín Federico García Arteaga

Tabla N³. Programa de titulación del ensayo de tratamiento a objetivo para la insulina basal en pacientes con diabetes mellitus

\begin{tabular}{|c|c|}
\hline Nivel de glucosa en ayunas. & Aumento de la insulina basal. \\
\hline 120 a $140 \mathrm{mg}$ por dL $(6.66$ a $7.77 \mathrm{mmol}$ por $\mathrm{L})$ & 2 unidades \\
141 a $160 \mathrm{mg}$ por dL $(7.83$ a $8.88 \mathrm{mmol}$ por $\mathrm{L})$ & 4 unidades \\
161 a $180 \mathrm{mg}$ por dL $(8.94$ a $9.99 \mathrm{mmol}$ por $\mathrm{L})$ & 6 unidades \\
$>180 \mathrm{mg}$ por dL $(9.99 \mathrm{mmol}$ por $\mathrm{L})$ & 8 unidades \\
\hline
\end{tabular}

Fuente: (Riddle, 2003).

Técnica de inyección de insulina

La insulina es efectiva solo si se administra adecuadamente. Se pueden administrar inyecciones en el abdomen, la parte externa del muslo, la parte posterior del brazo y la región del flanco-glúteos. La aguja debe colocarse en un ángulo de 90 grados con respecto a la piel y mantenerse en su lugar durante cinco a 10 segundos después de la inyección para evitar fuga de insulina. (ADA, 2004)

La rotación de los sitios de inyección es importante para prevenir la lipohipertrofia (es decir, tejido cicatricial de inyecciones repetidas en la misma área). La lipohipertrofia conduce a una pobre absorción de insulina y formación de depósitos, que pueden liberar insulina al azar, causando hiperglucemia posprandial temprana y / o hipoglucemia tardía.

La insulina está disponible en bolígrafos y viales. Los beneficios de las plumas de insulina incluyen la conveniencia de almacenar a temperatura ambiente durante 28 días después de la 


\section{Insulinoterapia en pacientes con diabetes mellitus tipo 2}

Vol. 3, núm. 2., (2019)

Kevin Horacio Illescas Ochoa; Cindy Mireya Castro Sánchez; Melba Katiuska Carrera Saltos; Martín Federico García Arteaga

apertura y la facilidad de uso para pacientes con problemas visuales o de destreza. Los pacientes con dificultades visuales pueden escuchar los "clics" de la pluma de insulina para contar el número de unidades. Se debe indicar a los pacientes que ceben la pluma de insulina antes de cada uso. El cebado consiste en extraer 1 o 2 unidades de insulina e inyectar en el aire para permitir que la insulina llene la aguja. (ADA, 2004)

\section{Conclusiones}

Durante el desarrollo de la investigación se observó que la terapia con insulina se recomienda para pacientes con diabetes mellitus tipo 2 y un nivel inicial de A1C mayor que 9 por ciento, o si la diabetes no está controlada a pesar de la terapia glucémica oral óptima. La terapia con insulina puede iniciarse como aumento, comenzando con 0.3 unidades por $\mathrm{kg}$, o como reemplazo, comenzando con 0.6 a 1.0 unidades por $\mathrm{kg}$.

También se observó que cuando se usa reemplazo en la terapia, el 50 por ciento de la dosis diaria total de insulina se administra como basal y el 50 por ciento como bolo, dividido antes del desayuno, el almuerzo y la cena. La terapia de aumento puede incluir insulina basal o en bolo. La terapia de reemplazo incluye insulina de bolo basal y corrección o insulina premezclada.

El control de glucosa, efectos adversos, costo, adherencia y la calidad de vida deben tenerse en cuenta al elegir la terapia. La metformina debe continuarse si es posible porque se ha demostrado que reduce la mortalidad por todas las causas y los eventos cardiovasculares en pacientes con diabetes con sobrepeso. 


\section{Insulinoterapia en pacientes con diabetes mellitus tipo 2}

Vol. 3, núm. 2., (2019)

Kevin Horacio Illescas Ochoa; Cindy Mireya Castro Sánchez; Melba Katiuska Carrera Saltos; Martín Federico García Arteaga

Finalmente se puede concluir, que la valoración de la insulina a lo largo del tiempo es crítica para mejorar el control glucémico y prevención de complicaciones relacionadas con la diabetes.

\section{Bibliografía}

ADA. (2004). Asociación Americana de Diabetes. Administración de insulina. Cuidado de la diabetes.

ADA. (2010). American Diabetes Association. Standards of medical care in diabetes. (págs. 11161). Diabetes Care.

Bolívar, J. (2015). Investigación Documental. México. Pax.

California, U. d. (10 de diciembre de 2010). Educación sobre diabetes en línea. Universidad de California, San Francisco. Obtenido de http: // www.deo.ucsf.edu.

Castro, J. (2016). Técnicas Documentales. México. Limusa.

Cobry, \& McFann. (2010). Momento de los bolos de insulina de comida para lograr un control glucémico posprandial óptimo en pacientes con diabetes tipo 1. Diabetes Technol Ther. , 173-177.

Davila, A. (2015). Diccionario de Términos Científicos. Caracas: Editorial Oasis.

Endotext.org. (6 de diciembre de 2010). El manejo de la diabetes tipo I. Recuperado el 9 de agosto de 2019, de http://www.endotext.org/diabetes/diabetes17/diabetesframe17.htm

Holman, \& Farmer. (2009). Three-year efficacy of complex insulin regimens in type 2 diabetes. Engl J Med, 361-406.

Jellinger, \& Davidson. (2007). Mapas de ruta para lograr el control glucémico en la diabetes mellitus tipo 2: Grupo de trabajo del mapa de ruta de diabetes ACE / AACE. Bogota: Endocr Pract.

Jonasson, \& Ljung. (2009). Uso de insulina glargina e incidencia a corto plazo de enfermedades malignas: un estudio de seguimiento basado en la población en Suecia. Diabetologia., (págs. 1745-1754.). Suecia. 


\section{Insulinoterapia en pacientes con diabetes mellitus tipo 2}

Vol. 3, núm. 2., (2019)

Kevin Horacio Illescas Ochoa; Cindy Mireya Castro Sánchez; Melba Katiuska Carrera Saltos; Martín Federico García Arteaga

Lanceta. (2010). Control intensivo de glucosa en sangre con sulfonilureas o insulina en comparación con el tratamiento convencional y el riesgo de complicaciones en pacientes con diabetes tipo 2. Grupo de Estudio Prospectivo de Diabetes. Reino Unido.

Lanceta. (2008). control intensivo de glucosa en sangre con metformina sobre las complicaciones en pacientes con sobrepeso y diabetes tipo 2 .

Miser, \& Arakaki. (2010). Evaluaciones aleatorias, abiertas y de grupos paralelos de la terapia de bolo basal versus terapia premezclada con insulina lispro en pacientes con diabetes mellitus tipo 2 que no logran el control con el tratamiento de insulina de inicio y los fármacos.

Riddle, R. (2003). El ensayo Treat-to-Target: adición aleatoria de glargina o insulina NPH humana a la terapia oral de pacientes con diabetes tipo 2. Cuidado de la diabetes.

Ritzel, \& Bulter. (2002). Physiology of glucose homeostasis and insulin secretion. Insulin Therapy. New York. Marcel Dekker, 61-72.

Singh, \& Ahmad. (2009). Efficacy and safety of insulin analogues for the management of diabetes mellitus: a meta-analysis.

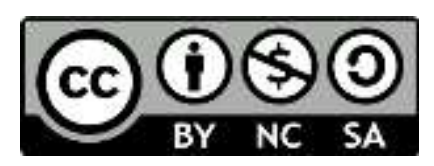

RECONOCIMIENTO-NOCOMERCIAL-COMPARTIRIGUAL

CC BY-NC-SA

ESTA LICENCIA PERMITE A OTROS ENTREMEZCLAR, AJUSTAR Y CONSTRUIR A PARTIR DE SU OBRA CON FINES NO COMERCIALES, SIEMPRE Y CUANDO LE RECONOZCAN LA AUTORÍA Y SUS NUEVAS CREACIONES ESTÉN BAJO UNA LICENCIA CON LOS MISMOS TÉRMINOS. 